latter to the initial transient polymyositis, if, indeed, the dystrophies are at all myositic in origin.

As a result of my own observations, I feel like seconding Gowers when he remarks that "it seems, therefore, undesirable to form a separate variety of juvenile muscular atrophy, as Erb has proposed. Spinal atrophies may also be juvenile." So also may myositic atrophies. 100 State Street.

\section{PREJUBERCULAR CONDITIONS AND THE TREATMENT OF ASSOCIATED ANEMIA BY HYPODERMIC INJECTIONS OF IRON AND ARSENIC.*}

B. R. SHURLY, MID.

Lecturer on Laryngology, Detrolt College of Medicine; Laryngologist, Harper and Childrens' Free Hospitals; Attending Physician, Woman's Hospital.

DETROIT.

It is a well-recognized fact that pulmonary tuberculosis is the cause of more deaths than any other one disease, except pneumonia. In the state of Michigan our mortality record for some years has averaged above 2,500, while in Detroit our last report shows that 327 individuals died from this cause. We are all familiar with the fact that nearly one-seventh of all persons die of tuberculosis, and more than 50 per cent. of the remainder give postmortem evidences of tuberculous foci that had remained dormant or succumbed entirely to the antitoxic properties of our cells.

If this latter astounding and well-conceded fact is true, seven out of ten individuals that reach late adult life have been infected with tuberculosis.

The profession and the laity are awakened to the stu. pendous problem at hand. Many of us appreciate our iuefficiency in battling with the ravages of this disease. We are especially apt to become therapeutic nihilists in the treatment of tuberculosis and to keep most prominently in mind the phenomena of hopeless advanced cases where all therapy has proved unsuccessful. It is with a hope of re-establishing an enthusiasm along the brighter and more encouraging fields of so-called pretubercular and early tuberculous conditions that I present this subject for your consideration.

Our daily routine work in the examination of the chest will divide our patients into five distinct degrees of disease.

First.-The acute miliary or the advanced chronic cases of pulmonary tuberculosis, generally incurable in any climate.

Second.-The moderately severe tuberculosis, a considerable number of whom will recover under proper treatment.

Third.-Early or incipient phthisis, 70 per cent. of whom it is estimated can be cured under favorable conditions.

Fourth.-Bronchopneumonitis and other non-tubercular lung diseases.

Fifth.-Cases with hereditary or acquired predisposition presenting prominent nutritional or constitutional changes, which we may classify for convenience as pretubercular conditions.

Fvery consumptive of the moderately or advanced type is putting up a fight for life, and we are helping lim as best we can. The symptoms of hemoptysis or the chills and fever of the mixed infections are usually signals for a change of physicians or a consultation.

- Read before the Wayne County (Mich.) Medical Society.
It is at these times that the relatives and the physicians become thoroughly alarmed, and no financial or physical effort can be too great to satisfy the anxious minds at these crises.

A most conclusive demonstration of the ravages of the disease up to the time of hemoptysis or when the evidences of mixed infection become pronounced is offered us by the $x$-ray. 'Two facts are at once apparent. First, phthisis pulmonalis progresses to small cavity formation usually before ordinary skill in percussion and auscultation reveals it; second, $x$-ray examination at the perion of hemoptysis or at the onset of fever frequently demonstrates extensive tubercular disease all out of proportion to the physical signs. A keen knowledge of the diagnosis and treatment of the so-called pretubercular stage, which antedates outward tubercular manifestations, is exceedingly important from all points of view.

Those who take careful histories of chest cases and carefully analyze a series of them will most certainly be impressed with the great number of persons who report a prevailing impoverishment or morbid state of the whole system for a considerable period before the apparent development of the local lesions. 'The general practitioner finds a deep problem in the relations of hereditary and acquired predisposition to pulmonary tuberculosis, and his therapeutic indications must be promptly met.

If I were to state that pretubercular phthisis pulmonalis was as distinctly a stage of phthisis as phthisis incipiens, with prominent fixed diagnostic signs and manifestations, I am confident many would assent. How many of us vigorously supply the therapeutic indications in this curable onsetting stage of tuberculosis? Are we justified in terming this condition tuberculosis from postmortem findings in individuals of this type? No, but the patients should have the benefit of our suspicions. It is impossible to overestimate the value to the patient or the state of timely applied hygiene and treatment in the pretubercular stages.

Some very interesting questions arise along this line of thought. First, where does the so-called pretubercular stage of phthisis end and incipient phthisis begin? Second, what methods of diagnosis have we that differentiate the stages and what treatment must be applied? Third, how much tuberculosis of the lungs may exist before ordinary skill in physical diagnosis can detect it? If we can successfully thresh out these problems and throw the brunt of our efforts on patients exhibiting the phenomena of the pretubercular or the incipient stages, the mortality from consumption would certainly be lowered. The maxim of Tyndale is certainly logical: "that localized tuberculosis does not endanger life of itself so long as the general nutrition begets a reasonable resisting power of the pulmonary tissues." And especially the pulmonary gland tissues, I might add. This is demonstrated to us daily in the postmortem findings in accidental deaths.

We may divide our tuberculous cases into two grand classes. First, the infections that have taken place in strong healthy individuals, who have no evidences of predisposition, acquired or hereditary, such as renowned athletes or prize cattle. Second, those cases which present a prodromal period of impaired general condition from all causes. It is this latter class that $I$ wish to consider.

It is my belief that the pretubercular stage of phthisis, as we now classify it, is, in the vast majority of cases, nothing more than a latent unrecognized tuber- 
culosis, and the development of the bacillus tuberculosis in many is a problem in cell nutrition, the biochemic phenomena of which we do not understand. The examination of recruits for the armies and navies of the world, performed by men eminently fitted for this work, reveals the fact that many cases of latent or incipient tubereulosis are admitted. The French army shows a record of 7.2 per cent. who soon develop phthisis, while the percentage is higher in the Austrian, German and American armies.

In the light of our present knowledge, we are unable to draw sharp lines of distinction between the pretubercular and incipient stages. There is no tuberculosis without the tubercle bacilli. It is the province of this paper, then, to classify all phenomena that predispose or lead up to the actual demonstration of incipient phthisis. For convenience in arrangement I have adopted and modified the classification of Loomis:

1. Corpulence, viz, the relation of the body weight in pounds to height in feet.

2. Chest conditions. A-Conformation of the Chest. BChest measurements. C-Vital capacity.

3. Constitutional conditions. A-Lymphatism. B-Digestive disturbances. C-Chloro-anemia.

4. Character of pulse.

Allow me to exclude from consideration the factors of sex, age, race, previous or present disease of the lungs, septicemia or other thoroughly appreciated etiologic factors.

Corpulence is taken from the French, and it is used to express mathematically any departure from a standard of ratio between weight and height. According to the military tables, a man, at height five feet eight inches, should weigh 150 pounds, having an average corpulence of $26.4 \%$. The ratio shown by dividing the weight by the height is $261 / 2$ as a standard. We appreciate entirely the important significance in progressive loss of weight without a demonstrable cause as a very constant symptom of onsetting phthisis. This fact of a continued loss in body weight is usually recognized as of importance only when it is followed by a hacking dry cough some months later. Accurate scales and a measurement of height in this class of patients are much more necessary than is generally admitted. A record is certainly as valuable in determining the nutrition of tuberculosis as the weight chart is in infant feeding. A majority of my patients have given a history of steadily increasing loss of weight before the lesions were diagnosed. Loomis reports 50 per cent. in a series of forty cases. Many thousand military observations have established a normal standard of corpulence for men as 26 , while the observations of Bonchard show that women should have a normal of 23 . A corpulence of 21 is considered abnormally thin.

Associated with steadily increasing loss of tissue is the prominent symptom of general malaise. The daily routine is marked by a substitution of will power for natural automatic power. We may note increasing motor debility, the patient may complain of being "sawed off at the legs," or a weakness in the knees. Sensations become abnormally accentuated or dulled; sleep becomes broken or profound; digestion, changeable respiration, superficial and slightly hurried, as 22 or 24 . Many cases show acceleration of the heart with relative falling off of arterial pressure. When these symptoms of disturbance are present, and after the exclusion of acute and chronic diseases, we thoroughly examine the chest and nothing definite can be determined; a true latent tubercular condition exists.
When this complex of symptoms arise, we should give additional attention to the predisposing group of conditions previously enumerated. We are well aware that a single tubercle requires two to four weeks in its process of evolution, and the foci must break down and infect often numerous areas before clinical symptoms appear. Let us consider, further, what danger signals of a possible onsetting tuberculosis are reliable aids to diagnosis.

Our insurance companies are particularly interested in all observations of dangerous chest conditions. We are well aware that there may be many deviations from the normal standard of chest conformation without in any special manner increasing the liability to phthisis. The postures of childhood or occupation may lead to faulty conformations without increasing the tendency to the invasion of the tubercle bacilli. Our suspicions are aroused when we have presented for examination the lean, hollow chest, with marked subclavicular depressions, with prominent spaces between the ribs, with projecting scapulæ, and decided diminution of the antero-posterior diameter.

Considerable and more valuable evidence may be obtained by scientific measurement of a chest. French military observers have placed much importance on what is called a determination of "the vigor of constitution." It is taken successfully in this manner: "Two ordinary measuring tapes are sewed together and the point of juncture rests in the center of the spine. In men the estimations are made at the nipple level, while in women the line of the ensiform cartilage is preferred. The double tape aids in measuring the difference in expansion of the right and left sides, as well as the total chest mobility. The right is usually at least a half-inch larger, although both lungs should show almost equal expansion." A circular measurement below 35 inches is abnormal. The average of measurements taken at the end of forced expiration and at the moment of forced inspiration is defined as the thoracic perimeter. The vigor of constitution is the relation between the perimeter and the height. The thoracic perimeter of a person, according to the French, should never be lower than one-half the height.

The question of vital capacity is important, but the deductions are more subject to error. Considered from its own standpoint alone, it is of litle value. Much depends on the previous education of the patient and the experience which the physician may have had in the use of the spirometer. When considered in relation to age, weight and particularly height, important data on respiratory capacity may be obtained. Standards have been made from a great number of cases, and we find that the relation between the height and the capacity should be one to three inches in men and one to two and six-tenths in women. A man of five feet eight inches should not fall under $204 \mathrm{cu}$. inches. The interrelation of corpulence, thoracic perimeter and vital capacity should be considered valuable aids to a determination of genuine predisposition to pulmonary tuberculosis.

We are well aware that pneumonia, pleuritis, infectious diseases, occupations, direct exposure, etc., are the great etiologic factors, but these are eliminated, as I have previously stated, because they are centers of infection from local causes that can be demonstrated. They do not constitute the true pretubercular conditions. The pretubercular stage in some individuals is marked by a constitutional condition called lymphatism. Pathologic arlenoids, tonsils, or hypertrophies interfere 
with nasal and nasopharyngeal drainage, the cervical lymphatic chains are taking in septic material and the bronchial glands are involved in like pathologic processes. A latent tuberculosis which it is impossible to diagnose may exist at this time or this type of glandular hypertrophy may take on infection and become a glandular tuberculosis of the lungs.

Another class of individuals may show a series of digestive disturbances prior to tubercular infections. There is a genuine relation between so-called indigestion that exists for months or years antecedent to phthisis. The malnutrition attending disorders or digestion renders the system much more vulnerable to invasion by the bacilli. Over-indulgence in indigestible food has increased this symptom.

It is especially the relation of chloroanemias to the development of phthisis that I wished to discuss and present for your consideration. Some investigators have claimed that an actual antagonism exists between tuberculosis and chlorosis. Some consider chlorotics well under way in the destructive processes of phthisis. However this may be, we can certainly be impressed with the constant attendance of anemic blood changes in cases that show imperfect chest development and continuous loss of weight for which no cause is apparent. It is especially a danger signal in men of tubercular age. Chlorosis ordinarily is not accompanied by loss of weight nor abnormal chest conditions. While the examination of the blood in the late stages of consumption is of no practical value, except that the absence of eosinophiles may be looked on as an unfavorable prognostic sign, examinations in the pretubercular conditions all show that the hemoglobin is diminished out of all proportion to the loss in red cells. Henocque, an extensive observer, says that a diminished supply of oxyhemoglobin characterizes tubercular chloroanemia. The hemoglobin never falls as low as in chlorosis. Early phthisis is usually marked by a slight leucocytosis. slight diminution of the red blood corpuscles, and a moderate reduction in hemoglobin.

Laache has found 29 per cent. of tubercular chloroanemias in the early stages with a diminished hemoglobin. Labbe, who has investigated extensively, regards chlorosis as a symptom of some other affection often as a first manifestation of a latent tuberculosis. If we should consider chlorosis or the simple anemias just as a pleuritis to be many times nothing more than the initial evidence of a slumbering tuberculosis, the proper hygienic and medicinal treatment can produce a complete recovery. A number of cases of anemias showing with many of the other symptoms of impaired resistances enumerated have come under my observations during the last year or two. The method of treatment has proved so successful that the plan may be of interest.

I have selected only a few cases as a preliminary report, as it is necessary that six months or a year should elapse before we can determine our results. Even the therapeutic nihilist will admit that iron, arsenic and the hyphophosphites have a wide field of usefulness, and as these are used hypodermically the effects are more pronounced, more rapid and permanent. Wherever iron is indicated, it can be administered in the form of the green ammoniated citrate, while arsenic can be given as the arsenate of soda or the arsenate of iron. The injections are given deeply into the muscles of the buttocks or back. There is almost no pain attending the operation and in indicated cases the general feeling of well-heing which follows the proper dosage allows the treatment to speak for itself. A blood examination should be made, after which punctures may be given daily. An increase of 5 to 10 per cent. hemoglobin a week can be expected.

It is unnecessary to consume time with a consideration of the therapeutic action of these substances. Nothing, however, has been written in English on the subject of the hypodermic use of these medicinals. Italian and French literature since 1890 has had much to say on the method, and the fact that it remains to-day as the usual treatment is sufficient recommendation for its usefulness.

The grcen ammonio-citrate can be introduced into the system without danger in doses ranging from .05 to $.1 \mathrm{gram}$, while the arsenate of soda is given in .001 to $.002 \mathrm{gram}$. The treatment should be started with the smaller dosage. A full dose of iron by this method produces a reaction within five minutes. A feeling of tension in the head is experienced, the face flushes and tingling sensations are noticed. There may be waves of nausea or sudden vomiting if a larger dose than $11 / 2$ grain is administered. A full dose gives a sensation of a warm flush or glow over the entire body. The pulse quickens and a general feeling of well-being follows the proper dose. The arsenate shows its usefulness as a general reconstructive and stimulant to nutrition. The hypodermic method obviates all injury to the teeth or stomach and constipation is not produced. It is contraindicated in fever and active hemorrhage. Iron and arsenic are too well known to need any argument in their favor. The hypodermic method produces all the good effects more promptly and without the serious results produced when these remedies are taken by the stomach.

The character of the pulse in determining the pretubercular stage of consumption is quite important. 1)r. Wells, of Chicago, and Dr. H. P. Loomis have made excellent reports in regard to their findings along this line. Two prominent characteristics are noted. A change in position has but little influence on the very thin. The examination must be made some hours after eating and when the patient is free from excitement. Ordinarily the pulse is found to vary on an average of fifteen beats in a healthy man. I am unable to make practical use of this observation, as few patients under cxamination are not subject to the pulse effects of excitement. The relative feebleness of arterial pressure is an important sign. The normal arterial pressure of $\mathbf{1 5}$ to 18 is found by French investigators to be always less than thirteen and often only ten. The lessened arterial pressure may be nothing more than a factor in the problem of increased susceptibility. The pulse of lowered pressure is usually tense and hurried, 100 to 120 . In the early stages of phthisis the sphygmograph shows a sudden ascent and descent of the curve. The arterial pressure can be readily taken in the office with a Gärtner's or preferably a Cook's pressure apparatus.

In conclusion, it is evident that no one or two signs can be relied on as conclusive proof of an approaching phthisis. Yet, taken together, we are given enough danger signals to warn us. Active measures may be enforcen at this stage with the best results. If the strenuous fight against the ravages of tuberculosis was advanced to the latent tubercular stages, the stages of lowered resistance, consumption would not attain its high mortality. Hypodermic medication with iron, arsenic, hyphophosphites and strychnin offers a prompt and powerful reconstructive adjunct to the necessary pure air, good food and sensible hygiene. 